\title{
Teoría euclidiana de la proporción en la construcción de los números reales: ¿un asunto útil para un profesor?
}

\section{Edgar Alberto Guacaneme Suárez*}

Artículo recibido: 30-08-2010 y aprobado: 04-05-2012

\section{Euclidean Theory of Proportion in the Construction of Real Numbers: A Useful Subject for a Teacher?}

Abstract: From the Greek Golden

Resumen: Desde la época dorada griega, la teoría euclidiana de la proporción, expresada en el Libro V de Elementos, se constituyó en esquema para la formulación de relaciones entre magnitudes, sin interesar si estas eran o no conmensurables $y$, en consecuencia, sin recurrir a los valores numéricos de sus medidas para establecer tanto las razones entre magnitudes, como la proporción entre razones. Cerca de veinte siglos después, esta manera de tratamiento independiente de una estrategia aritmética parece ser precisa y, paradójicamente, el acicate y guía para la constitución del conjunto de números reales. Los historiadores de las Matemáticas han discutido la relación entre estas teorías y conjeturamos que el estudio de sus posturas puede traer beneficios a la educación del profesor de Matemáticas. Estos beneficios se refieren, entre otros aspectos, a visiones alternas de la actividad matemática de estudio de una teoría y a la ampliación de la mirada sobre los objetos matemáticos implicados en las teorías.

Palabras clave: Razón, proporción, número real, Euclides, Dedekind, Frege, conocimiento del profesor.
Age, the Euclidean theory of proportion, stated in Book $\mathrm{V}$ of Elements, was established as an outline for the formulation of relations between magnitudes, were they or not measurable and, therefore, without resort to numerical values of its measurements to establish both the ratios of magnitudes as the proportion between ratios. Nearly twenty centuries later, this way of independent treatment of arithmetic strategy seems to be accurate and, paradoxically, the incentive and guidance for the establishment of the set of real numbers. Historians of mathematics have discussed the relationship between these theories and we conjecture that the study of their positions can bring benefits to the mathematics teacher education. These benefits relate with alternative visions of the mathematical study of a theory and the extension of the look on the mathematical objects involved in theories.

Keywords: Ratio, proportion, real number, Euclid, Dedekind, Frege, teacher's knowledge.

\footnotetext{
* Profesor del Departamento de Matemáticas de la Universidad Pedagógica Nacional. Estudiante del Doctorado Interinstitucional en Educación - Énfasis en Educación Matemática (Sede Universidad del Valle). guacaneme@pedagogica.edu.co
} 


\section{Introducción}

En el intento de hacer una aproximación a la historia de los conceptos de razón y proporción, desde nuestra posición de aprendices de la historia $-\mathrm{y}$ no de historiadores-, hemos llevado a cabo una búsqueda bibliográfica que nos ha conducido a identificar más de un centenar y medio de escritos de historiadores de las matemáticas y filósofos de las ciencias, que versan sobre dicho asunto. Aun sin haber hecho un estudio profundo de todos esos documentos, nos atrevemos a afirmar que la gran mayoría de ellos tratan momentos y hechos específicos del desarrollo de estos conceptos y exhiben análisis diversos sobre los mismos; esta especificidad contrasta con la ilusión -ingenua y hasta romántica-que teníamos al iniciar la búsqueda bibliográfica de encontrar una historia de la evolución de los conceptos de razón y proporción.

A través del estudio de algunos de los documentos identificados, hemos podido perfilar varios hitos que, de manera secuencial, describen varias épocas de la historia de los conceptos de razón y proporción, a saber:

a. La época de la escuela pitagórica, en la cual existía una teoría de las proporciones que, según la tradición hegemónica', entra en crisis por el "descubrimiento" de la inconmensurabilidad;

b. la época dorada de los griegos (fundamentalmente de Eudoxo, Euclides y Apolonio) en la que se "crea" una teoría de las pro-

1 Esta tradición ha sido cuestionada por autores como Knorr (2001). porciones, se adapta a la versión hipotético-deductiva y se usa en la descripción de las cónicas;

c. la época del surgimiento de lo que hoy se llama álgebra $-\mathrm{y}$ particularmente de la geometría analítica- en la que se hace uso de la teoría de las proporciones en la solución de problemas geométricos, a través de procedimientos analíticos;

d. la época del Renacimiento, en la que la clásica teoría de proporciones griega se transforma y reformula para ampliar su ámbito de aplicación a magnitudes no geométricas y su empleo en las ciencias naturales y médicas;

e. la época de creación del Cálculo y del Análisis, en la que el lenguaje de las funciones sustituye el clásico lenguaje de las proporciones empleado por varios siglos, cayendo este último en un estado de aletargamiento;

f. la época de desarrollo de los trabajos del matemático alemán Julius W.R. Dedekind y del matemático y filósofo alemán Gottlob Frege, relativos a la construcción del conjunto de los números reales, en la que de manera un poco intempestiva, la teoría euclidiana parece renacer en cuanto a su protagonismo, como acicate en dichas construcciones.

De estos hitos hemos estudiado, con entusiasmo y relativa intensidad, varios resultados de la investigación y reflexión de la historia de la Matemática acerca de la teoría euclidiana de la proporción presentada en el Libro V de Elementos 
(Guacaneme, en prensa), y actualmente investigamos sobre el potencial formativo que algunos de tales resultados tendrían en la educación del profesor de Matemáticas.

Colateralmente $-\mathrm{y}$ mediado por un interesante planteamiento de GrattanGuinness (2004a, 2004b) acerca de la posibilidad de estudiar las "consecuencias históricas" de objetos matemáticos, desde al menos dos maneras de aprehender las matemáticas del pasado- hemos abordado el estudio de una problemática vinculada al último de los hitos reseñados antes. Esta se expresa en diversos documentos de la bibliografía consultada, a través de textos que aluden a una cierta analogía entre la definición 5 del Libro V de Elementos y la definición de los números reales expresada por Dedekind. Varias de las referencias presentan esta analogía como una cierta identidad de la razón de dos magnitudes con un número; veámoslas a través de la traducción que hemos hecho al español, cuando ha sido necesario:

La razón de una magnitud $A$ a otra magnitud $B$ de la misma clase es un número real, racional o irracional, determinado de la manera como se explican en lo que sigue. Este se denota por el símbolo A:B. (Hill, 1912, p. 360)

Estos seis resultados suministran una regla para determinar si la razón $A: B$ es mayor que, o igual a, o menor que, cualquier número racional; consecuentemente, en concordancia con la definición de Dedekind, la razón $A: B$ es considerada como un número. (Hill, 1928, p. 44)
Esto significa que la definición de número real propuesta por Richard Dedekind es la misma que la presentada por Eudoxio. (Zubieta, 1991, p. 478)

Otras, por su parte, presentan esta analogía como una correspondencia, interpretación o equivalencia:

...existe una correspondencia exacta, casi una coincidencia, entre la definición euclidiana de identidad de razones y la teoría moderna, debida a Dedekind, de los números irracionales. (Heath, 1956, p. 124)

Esta nota presenta la definición de número real atribuida a Dedekind como una interpretación de la definición de proporción de Eudoxio, tal como la enuncia Euclides al principio de su Libro V. (Zubieta, 1991, p. 477)

Como es bien sabido, las definiciones euclidianas son un equivalente de la técnica de Dedekind (a través de «cortes» en los racionales) para investigar la propiedad de los números reales. (Knorr, 1992, p. 3)

Otras, sencillamente, exhiben un tipo de relación de dependencia:

Después de más de dos milenios, la teoría de las proporciones de Eudoxio llegó a ser la base de la teoría de los números reales de Dedekind (que data de los años 1870). Aproximadamente, la teoría de las proporciones de 
Eudoxio corresponde a la teoría de los números reales (positivos) bajo multiplicación (Shenitzer, 1995, p. 286).

A continuación recapitularemos algunos asuntos que hemos explorado y estudiado sobre la relación entre la teoría euclidiana de la proporción y la construcción de los números reales. Inicialmente presentaremos algunos aspectos relevantes del Libro V de Elementos de Euclides, enfatizando en la idea de que en la teoría allí expuesta no es pertinente concebir las razones como números, ni la proporción como igualdad entre razones, ni operaciones entre razones. Luego, expondremos una mirada a la discusión en torno al uso que, al parecer, Dedekind hizo de la teoría euclidiana de la proporción; igualmente, a modo de hipótesis, arriesgaremos una interpretación de la manera como Frege pudo atender a aspectos de tal teoría. Finalmente, exhibiremos algunas conclusiones respecto de la intervención de la teoría euclidiana en la construcción de los números reales y presentaremos algunas reflexiones respecto de una posible utilidad de incorporar un estudio similar al presentado, en el marco de la educación de un profesor de Matemáticas.

\section{Algunos aspectos de la teoría de la proporción en el Libro $V$ de Elementos}

Si bien varios de los trece libros que componen Elementos, magistral obra de Euclides, abordan cuestiones sobre las razones de magnitudes geométricas o números y sobre las proporciones, hemos centrado nuestra atención en el Libro V, debido a que en este se exhibe la reelaboración euclidiana de la teoría de la proporción eudoxiana, que plantea un tratamiento general para las magnitudes geométricas y ha sido ampliamente estudiada por los historiadores y filósofos; además, es esta teoría $-\mathrm{y}$ no precisamente a la de la proporción numérica tratada en los Libros VII, VIII y IX - la que se puede vincular con los trabajos sobre los números reales de Dedekind y Frege.

Al explorar la configuración del Libro V, en la versión de Puertas (1994, pp. 9-54), se distinguen: un grupo de dieciocho definiciones, veinticinco proposiciones - ninguna de ellas construcción o problema - y dos porismas o corolarios. A continuación nos referiremos primero a algunas de las definiciones y posteriormente a una clasificación de las proposiciones.

\section{Interpretación de algunas definiciones del Libro V}

En su orden, las dieciocho definiciones procuran caracterizar y nominar las ideas de: parte, múltiplo, razón, guardar razón, guardar la misma razón, magnitudes proporcionales, razón mayor, menor proporción, razón duplicada, razón triplicada, magnitudes correspondientes, razón por alternancia, razón por inversión, composición de una razón, separación de una razón, conversión de una razón, razón por igualdad y proporción perturbada. El contenido de algunas de las definiciones contempla otras ideas no definidas en Elementos, tales como: magnitud, medir (o ser medido), tamaño, magnitudes homogéneas, equimúltiplo, comparación de magnitudes, antecedente, consecuente, extremos, medios, adición de magnitudes y diferencia de magnitudes. 
Ahora bien, la historia de la teoría euclidiana de la proporción ha prestado especial atención a las definiciones de razón, guardar la misma razón [proporción] y razón mayor [desproporción] (definiciones 3,5 y 7 , respectivamente). Veámoslas y discutámoslas brevemente una a una.

Definición 3: una razón es determinada relación con respecto a su tamaño entre dos magnitudes homogéneas. (Puertas, 1994, p. 9)

Existe un cierto consenso en que la definición 3 presenta un carácter general y vago, respecto de la intención de definir la idea de razón. Desde nuestra perspectiva, este carácter no constituye una falencia en la teoría y, por el contrario, devela que en el conjunto de la teoría euclidiana de la proporción, no es pertinente precisarla más.

No obstante esta postura, advertimos conveniente reseñar que las ideas de tamaño y magnitudes homogéneas, implicadas en la definición 3, ameritan ser clarificadas, incluso a través de una referencia a la intuición. Respecto del tamaño de una magnitud, se puede señalar que es una característica intrínseca del objeto geométrico, de orden cuantitativo, no numérico, y no de este en relación con otro; así, por ejemplo, el tamaño de un segmento es la cantidad de longitud del mismo, pero no la medi$\mathrm{da}^{2}$ de la cantidad de su longitud, o bien, el tamaño de una región es la cantidad de superficie de esta, pero no su área.

2 Número asociado a este, en relación con otro segmento unidad.
Respecto de la idea de homogeneidad entre las magnitudes, basta con señalar que ello refiere a que las dos magnitudes involucradas en una razón no pueden ser de diferente naturaleza (i.e., no se puede establecer una razón entre el tamaño de un segmento y el tamaño de una superficie, así como tampoco entre el tamaño de un ángulo y el tamaño de un volumen, entre otros).

Bajo esta perspectiva la definición 3, más allá de definir la idea de razón y aún sin precisar la idea de relación, condiciona parcialmente ${ }^{3}$ la posibilidad de su existencia y apuntala aspectos respecto de su naturaleza; particularmente descarta la posibilidad de entenderla como una relación entre números (pero sí entre tamaños de magnitudes) e incluso como un número.

El papel aparentemente secundario de la definición 3, contrasta con el papel central en la teoría que le adjudican los historiadores y filósofos a la definición 5:

Definición 5: se dice que una primera magnitud guarda la misma razón con una segunda que una tercera con una cuarta, cuando cualesquiera equimúltiplos de la primera y la tercera excedan a la par, sean iguales a la par o resulten inferiores a la par, que cualesquiera equimúltiplos de la segunda y la cuarta, respectivamentey tomados en el orden correspondiente. (Puertas, 1994, p. 11)

3 La definición 4 establece una condición adicional sobre las magnitudes para que pueda darse por supuesta la existencia de la razón entre estas. 
Su interpretación en primera instancia se dificulta, desde nuestra perspectiva y experiencia, por la falta de apropiación del lenguaje retórico en que está escrita - o la carencia de un lenguaje simbólico o gráfico que la acompañe y represente-, por la vaguedad de la idea de equimúltiplo y por la generalidad implicada en la condición que se tiene que satisfacer para todos los equimúltipos (o mejor, para todas las parejas de equimúltiplos).

Para contrarrestar tal dificultad, recurramos al lenguaje gráfico a través de unos dibujos que representen las magnitudes implicadas, así: sean A y B segmentos, y C y D cuadrados, de los tamaños indicados en la Figura 1:

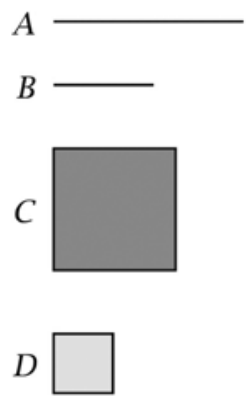

Figura 1. Cuatro magnitudes geométricas homogéneas dos a dos

Atendiendo a la particularidad connatural de los dibujos, construyamos los múltiplos dos de A y C así como los múltiplos tres de $\mathrm{B}$ y $\mathrm{D}$, obteniendo los dibujos que se muestran en la Figura $2^{4}$.

4 El múltiplo dos no es otro que una magnitud del doble del tamaño que el de la inicial de referencia; de manera análoga el múltiplo tres será una magnitud del triple de tamaño que el tamaño de la magnitud inicial de referencia. La construcción de los múltiplos se puede hacer a través del uso de regla y compás; para el caso de los cuadrados, se puede utilizar la proposición 47 del Libro I (o teorema de Pitágoras) como "algoritmo de la suma de cuadrados". múltiplo dos de A

múltiplo tres de $B$

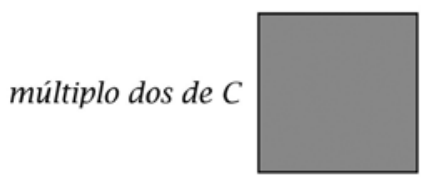

múltiplo tres de D

Figura 2. Múltiplos dos de A y C y múltiplos tres de B y D

Comparando ahora los tamaños del múltiplo dos de A con el del múltiplo tres de B y a la vez el tamaño del múltiplo dos de $\mathrm{C}$ con el múltiplo tres de $\mathrm{D}$, se establece que el múltiplo dos de A "es menor" que el múltiplo tres de B y a la vez el múltiplo dos de C "es menor" que el múltiplo tres de $\mathrm{D}$, existiendo entonces la posibilidad de que $A$ guarde con $B$ la misma razón que $C$ guarda con $D$. Si la comparación de los múltiplos de A y B arroja siempre el mismo resultado que la comparación de C y D (para los respectivos equimúltiplos), entonces se confirmará que A guarda con B la misma razón que $C$ guarda con $D$.

Para el ejemplo en cuestión se comprueba que las cuatro magnitudes no satisfacen la definición 5 o, en otras palabras, se verifica que A no guarda con $\mathrm{B}$ la misma razón que $\mathrm{C}$ con $\mathrm{D}$, pues luego de construir los múltiplos dos de A y C y los múltiplos cuatro de B y D se verifica que, si bien el múltiplo dos de A "es menor" que el múltiplo cuatro de B, el múltiplo dos de C "es mayor" que el múltiplo cuatro de D, como se observa en la Figura 3. 
múltiplo dos de A

múltiplo cuatro de B

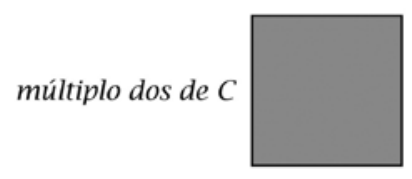

múltiplo cuatro de D

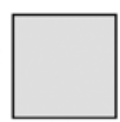

Figura 3. Múltiplos dos de A y C y múltiplos cuatro de B y D

Notemos desde ya que en el ejemplo no se requirió saber o precisar cuál era la relación (o razón) entre A y B, o cuál la relación (o razón) entre C y D para establecer que no guardan la misma razón.

Otra estrategia para intentar clarificar la interpretación de la definición 5 , es recurrir a la traducción simbólica del contenido de la definición a través de expresiones lógicas y algebraicas. En esta dirección, en los textos de los historiadores se reportan varias versiones simbólicas (no euclidianas, por supuesto); por ejemplo, Puertas (1994) reseña dos de tales versiones en las cuales $a, b$, $c, d$ son unas magnitudes del dominio de la teoría y $m$ y $n$ unos números naturales cualesquiera; en estas, respectivamente, se establece que se da una proporción $a: b:: c: d$ si y solo si:

$$
\begin{aligned}
& ((m \cdot a>n \cdot b) \text { y }(m \cdot c>n \cdot d)) \\
& ((m \cdot a=n \cdot b) \text { y }(m \cdot c=n \cdot d)) \\
& ((m \cdot a<n \cdot b) \text { y }(m \cdot c<n \cdot d)) .(p .12)
\end{aligned}
$$

(Si $m \cdot a>n \cdot b$, entonces $m \cdot c>n \cdot d)$ y (si $m \cdot a=n \cdot b$, entonces $m \cdot c=n \cdot d)$ y $($ si $m \cdot a<n \cdot b$, entonces $m \cdot c<n \cdot d) .($ p. 12)
Como se puede observar, la primera se formula como disyunción de conjunciones, en tanto que la segunda es expresada como conjunción de condiciones (o conjunción de implicaciones) $)^{5}$. Más allá de la observación hecha por Puertas (1994, p. 12) sobre la no equivalencia lógica entre las dos fórmulas anteriores, resaltemos que en las expresiones simbólicas solo se incorporan relaciones entre múltiplos, pero no la razón entre dos de tales magnitudes.

Probablemente la interpretación de la definición 5 que se ha logrado a través de las representaciones gráfica y simbólica, expuestas antes, contrasta $-\mathrm{y}$ eventualmente se complementa- con la sugestiva interpretación que hace Fine (1917) y que hemos traducido al español:

Ya que según la Definición 5 la condición para que $A, B, X, Y$ sean proporcionales es que: si los múltiplos $A, 2 A, 3 A, \ldots$ y $B, 2 B, 3 B$, ... son dispuestos en un arreglo en una sola secuencia en el orden de tamaño, y de la misma manera se disponen los múltiplos $X, 2 X$, $3 X, \ldots$ y $Y, 2 Y, 3 Y, \ldots$, la ley de distribución de los múltiplos de $A$ entre aquellos de $B$ debe ser la misma que la de los múltiplos de $X$ entre aquellos de $Y$. De ahí que «la identidad» de las razones $A: B$ y $X: Y$ significa la identidad de estas dos leyes de distribución, y la razón $A: B$ en sí misma significa la relación de tamaño entre $A$ y

5 A través de una notación particular de la reunión de los signos "=", "<" ${ }^{\prime \prime}>$ ", Filep (2003, p. 1) incorpora una forma simbólica equivalente a la segunda citada. 
$B$ que es indicada por la manera en que los múltiplos de $A$ están distribuidos entre aquellos de $B$. (p. 73)

Como se aprecia, esta interpretación alude a una correspondencia entre dos sucesiones ordenadas de múltiplos, cada una compuesta por los múltiplos de las dos magnitudes de una razón. Además, Fine sostiene que para una teoría general de la proporción no se requiere una definición de razón, en singular, aunque sí se exigen sendas definiciones de igualdad y desigualdad entre razones, las cuales son respectivamente proveídas en las definiciones 5 y 7 (guardar la misma razón y razón mayor). Veamos esta última.

Definición 7: entre los equimúltiplos, cuando el múltiplo de la primera excede al múltiplo de la segunda pero el múltiplo de la tercera no excede al múltiplo de la cuarta, entonces se dice que la primera guarda con la segunda una razón mayor que la tercera con la cuarta. (Puertas, 1994, p. 13)

Haciendo uso de la Definición 7 para el ejemplo gráfico que planteamos antes como interpretación de la Definición 5, se tendría que la razón que guarda $C$ con $D$ "es mayor" que la razón que guarda $A$ con $B$, en tanto que, como señala Fine (1917, p. 73) al parafrasear la Definición 7 , se logró encontrar $m$ y $n$ tal que

$m \cdot \mathrm{A}<n \cdot \mathrm{B}$ pero $m \cdot \mathrm{C}>n \cdot \mathrm{D}$.

Con respecto a lo planteado por los historiadores sobre la Definición 7 , resaltemos que Knorr (1992) establece que:
Uno de los defectos conocidos de la teoría euclidiana en el Libro $V$ es que no prueba que «no tener la misma razón» sea equivalente a «tener una razón mayor o menor razón»(p. 8)

Sin embargo, al explorar el uso que se hace de esta definición en la teoría (por ejemplo, en el Libro V, proposiciones 9 y 10), se corrobora que Euclides sí supone tal equivalencia, con lo cual dispone de una herramienta potente para la demostración de la proporción o desproporción de cuatro magnitudes; en otras palabras, si se supone que tener una razón mayor que otra equivale a afirmar que no es cierto que exista proporción entre tales magnitudes, se dispone de una herramienta para demostrar por reducción al absurdo.

En el marco de las interpretaciones y observaciones relatadas antes para las definiciones consideradas, hemos querido dejar claro, desde nuestra perspectiva e interpretación, en la teoría euclidiana de la proporción contenida en el Libro V:

a. Las razones son relaciones, pero no son números ni se establecen entre números, sino entre tamaños de magnitudes geométricas homogéneas dos a dos.

b. La proporción no es una igualdad entre razones (y por tanto no es igualdad entre números) ni depende de estas, sino fundamentalmente del comportamiento relativo y correspondiente de los múltiplos de las magnitudes implicadas. 


\section{Una clasificación de las proposiciones y porismas del Libro V}

Las veinticinco proposiciones y los dos porismas se pueden clasificar en cinco grupos de propiedades: cuatro de ellos atienden a los diferentes dominios que relaciona cada proposición (v.g., magnitudes y magnitudes, magnitudes y proporciones, proporciones y magnitudes, proporciones y proporciones) en tanto que el quinto ubica una proposición que no establece relación entre ninguno de tales dominios.

Así, en un primer grupo estarían las proposiciones que se refieren a las magnitudes y sus múltiplos, pero que no aluden a las razones ni a las proporciones. En este grupo hemos clasificado las proposiciones $1,2,3,5$ y 6 . Las expresiones simbólicas $\left[m\left(A_{1}+A_{2}+\ldots+A_{n}\right)=\right.$ $\left.m A_{1}+m A_{2}+\ldots+m A_{n}\right],[(m \oplus n) A=m A+n A]$, $[m(n A)=(m \cdot n) A],[m(A-B)=m A-m B] \mathrm{y}$ $[(m \ominus n) A=m A-n A]$ constituyen una interpretación simbólica de cada una de las proposiciones aludidas. Como se ve a través de estas - aunque también en la retórica enunciación euclidiana- no se incluye alusión alguna a la razón ni a la proporción, aunque sí a los múltiplos de las magnitudes, a la suma o diferencia de magnitudes, o a la adición, diferencia y multiplicación de los números ligados a los múltiplos. En cierto sentido, este conjunto de proposiciones caracteriza parcialmente el dominio de magnitudes con la operación suma.

En un segundo grupo ubicamos dos proposiciones ( $7-\sin$ su porisma 7 'y 8), que se pueden representar con sendas expresiones: [Si $A=B$, entonces $A: C:: B: C$ y $C: A:: C: B]$ y $[S i \quad A<B$, entonces
$A: C<B: C$; y $C: A>C: B]$, respectivamente. Una interpretación de estas expresiones permitiría reconocer que las proposiciones aluden a propiedades "de orden" de las razones a partir de propiedades “de orden" en las magnitudes; en otras palabras, estas proposiciones expresarían cómo la igualdad o desigualdad de las magnitudes se refleja o trasmite a algunas de las razones en que ellas están implicadas.

El tercer grupo está integrado por proposiciones $(9,10,14,20,21$ y 25) que describen cómo las relaciones entre razones determinan relaciones $\mathrm{u}$ operaciones entre magnitudes. Sus expresiones simbólicas respectivamente son: [Si $A: C: \because B: C$, entonces $A=B$. Y, si $C: A:: C: B$, entonces $A=B$ ], [Si $A: C<B: C$, entonces $A<B$. Y si $C: A<C: B$, entonces $A>B]$, [Si $A: B:: C: D$ y $A_{\gtrless}^{\gtrless} C$, entonces $B_{\gtrless}^{\gtrless} D$ ], [Si $A: B:: D: E$ y $B: C:: E: C$ y $A_{\gtrless}^{\gtrless} C$, entonces $D \gtrless$ [Si $A: B:: E: C$ y $B: C:: D: E$ y $A \gtreqless C$, entonces $D \gtrless C$ ], [Si $A: B:: C: D$ y $A>B$ y $A>C$ y $B>D$ y $C>D$, entonces $A+D>B+C$ ]. Una interpretación semejante a la hecha para el segundo grupo referiría que las proposiciones aluden a propiedades "de orden" de las magnitudes a partir de propiedades "de orden" de las razones.

El cuarto grupo contiene el mayor número de proposiciones (4, 7', 11, 12, $13,16,17,18,19,19$ ', 22, 23 у 24) е incluye propiedades de las proporciones o desproporciones, es decir, de las razones en sí mismas. Sus enunciados simbólicos respectivamente son: [Si $A: B:: C: D$, entonces para todo $m$ y $n$, $m A: n B:: m C: n D],[S i \quad A: B:: C: D$, entonces $B: A:: D: C$ ], [Si $A: B:: C: D$ y $C: D:: E: F$, entonces $A: B:: E: F],\left[S i A_{1}: B_{1}:: A_{2}: B_{2}:: \ldots\right.$ :: $A_{n}: B_{n}$, entonces $\left(A_{1}+A_{2}+\ldots+A_{n}\right):\left(B_{1}+B_{2}+\ldots+B_{n}\right)$ $:: A_{i}: B_{i}$, para todo $\left.i=1, \ldots, n\right]$ ], [Si $A: B:: C: D$ y 
$C: D>E: F$, entonces $A: B>E: F],[S i A: B:: C: D$, entonces $A: C:: B: D$ (a condición que las cuatro magnitudes sean homogéneas)], [Si $(A+B): B::(C+D): D$, entonces $A: B:: C: D]$, [Si $A: B:: C: D$, entonces $(A+B): B::(C+D): D$ ], [Si $(A+B):(C+D):: A: C$, entonces $(A+B):(C+D):: B: D],[\operatorname{Si}(A+B):(C+D):: B: D$, entonces $(A+B):(C+D):: A: C]$, [Si $A_{1}: A_{2}:: B_{1}: B_{2}$, $A_{2}: A_{3}: B_{2}: B_{3}, \ldots$, y $A_{n 1}: A_{n}:: B_{n 1}: B_{n}$, entonces $\left.A_{1}: A_{n}:: B_{1}: B_{n}\right],[S i \quad A: B:: E: F$ y $B: C:: D: E$, entonces $A: C:: D: F]$, [Si $A: B:: C: D$ y $E: B:: F: D$, entonces $(A+E): B::(C+F): D])$.

El último grupo contiene únicamente a la proposición 15, la cual solo alude a una proporción. Su expresión simbólica es simplemente $[A: B \% \circ A: n B]$.

Entre otros aspectos importantes, la anterior clasificación permite reconocer que no existen proposiciones que establezcan operaciones entre razones, a pesar de que sí se admitan operaciones entre las magnitudes implicadas en las razones. Este punto será trascendental en la argumentación que adelante explicitaremos respecto de la imposibilidad de reconocer en la teoría euclidiana una estructura para las razones, similar a la que se tendría para los números reales ${ }^{6}$.

\section{Dedekind y la teoría de la proporción}

En la mayoría de los tratamientos encontrados en los documentos de donde proceden las citas presentadas en la introducción de este documento $-\mathrm{y}$

6 Debemos precisar que nuestra postura contrasta con la presentada por Berghout $(1974,1975)$ a través de la cual, con una lectura moderna mediada por la identificación de estructuras algebraicas y de orden, identifica las proporciones euclidianas con los números reales. en otras que no hemos referenciado-, se encuentra la siguiente traducción simbólica ${ }^{7}$ de la definición 5 del Libro $\mathrm{V}$ de Elementos:

$A: B=C: D$ si y solo si para cualesquiera enteros positivos $n, m$,

Si $n A=m B$ entonces, $n C=m D$

Si $n A>m B$ entonces, $n C>m D$

Si $n A<m B$ entonces, $n C<m D$

Haciendo transformaciones algebraicas en las expresiones anteriores - sin necesariamente considerar su viabilidad y rigurosidad-, normalmente se obtienen las sentencias siguientes:

Si $A / B=m / n$ entonces, $C / D=m / n$

Si $A / B>m / n$ entonces, $C / D>m / n$

Si $A / B<m / n$ entonces, $C / D<m / n$

$\mathrm{Al}$ interpretar tales sentencias, se puede argüir que, para el primer caso (i.e., cuando las magnitudes $A$ y $B$ son conmensurables) la razón $A / B$ (o el número racional $m / n$, dado que son iguales) fragmenta al conjunto de los racionales en dos clases definidas, respectivamente, por los racionales mayores o menores

7 Nótese que esta no corresponde a ninguna de las reseñadas antes en este documento y que, respecto de aquellas, carece de varios conectivos lógicos.

8 La interpretación que hace Fine (1917, pp. 75-76) es un poco diferente a esta. En aquella incorpora una notación de parejas ordenas, similar a la que utiliza Dedekind en la exposición de sus cortaduras, lo cual hace más evidente la cercanía entre las dos teorías. 
que $m / n$, donde este pertenece a alguna de ellas. Para el segundo y tercer caso, la razón $A / B$ fragmenta al conjunto de los racionales en dos clases de números estrictamente mayores o menores que esta, en tanto que esta se da entre magnitudes inconmensurables y no puede ser expresada como un número racional. Desde una perspectiva general, la anterior interpretación es en esencia análoga a la que se puede hacer de la construcción de los reales presentada por Dedekind y recapitulada al español en una obra de divulgación de la historia de las Matemáticas (Dedekind, 1968).

Sin embargo, como lo menciona Leo Corry (1994, p. 8) ${ }^{9}$, existen historiadores de las matemáticas que rechazan la existencia de una correspondencia o equivalencia entre la teoría euclidiana de las proporciones y la teoría de los números reales de Dedekind, bajo la idea que la interpretación de los textos griegos debe hacerse en el marco de las restricciones en que estos fueron producidos.

Una de tales restricciones se refiere al lenguaje; en este sentido, no se acepta la traducción o formulación simbólica de las definiciones, ni las interpretaciones y deducciones que a partir de ellas se hacen. Otra de las restricciones obedece a la radical diferencia entre la idea de número de la obra griega y la del número del siglo XIX; la idea griega reconoce a los números y las magnitudes como cantidades no abstractas asociadas respectivamente al contar y medir, en

9 Debemos destacar el papel iluminador de este documento a favor de la comprensión de la no equivalencia entre la teoría euclidiana y la propuesta de Dedekind. tanto que la idea moderna se refiere a la cantidad como abstracta y general. En Elementos se establece una clara diferencia entre el contexto de las magnitudes geométricas (o cantidades continuas) y el de los números (o cantidades discretas), al punto que hay una formulación específica de teoría de la proporción en cada uno de ellos.

Más allá de la discrepancia interpretativa entre los historiadores, debemos reseñar que también se presentó entre el matemático alemán Rudolph Otto Sigismund Lipschitz y, precisamente, Julius Wilhelm Richard Dedekind. Lipschitz, en su correspondencia con Dedekind ${ }^{10}$, le manifestó que su teoría de las cortaduras difería tan solo en formulación, pero no en contenido matemático, de la concepción griega de número. De manera específica, Lipschitz rechazaba la afirmación de que tal teoría fuera la primera en demostrar legítimamente que $\sqrt{ } 2 \cdot \sqrt{ } 3=\sqrt{ } 6$.

Más allá de la preexistencia de tal prueba, para Dedekind estaba la pregunta si la teoría euclidiana contenía o no todas las suposiciones suficientes y necesarias para probar el teorema; su respuesta era un no rotundo, y su argumento reposaba en el hecho que aquella no contemplaba el principio de continuidad, médula de la teoría de cortaduras. El argumento de Dedekind aludía a las condiciones del dominio de las razones de magnitudes euclidianas y a la imposibilidad de definir apropia-

10 En la página http://www.uv.es/ jkliment/Documentos/Dedekind.pc.pdf está alojado un documento de traducciones al español de varios documentos de Dedekind, entre otros, su correspondencia con Lipschitz (pp. 15-25). 
damente operaciones aritméticas para estas. Tal argumento fue cuestionado por Lipschitz bajo el supuesto de que las magnitudes euclidianas son de por sí continuas y que por tanto no se requiere de la formulación explícita del principio de continuidad.

Esto fue discutido por Dedekind, quien apeló a la diferencia en intencionalidad de su trabajo con la del autor griego, a la naturaleza de otros supuestos en la teoría de la proporción contrastada con la del principio de continuidad, y a la naturaleza del espacio considerado por Euclides, y concluyó que la continuidad del espacio no era un supuesto que validara o invalidara el sistema euclidiano. De esta manera, Dedekind estableció en qué punto su teoría podía suministrar respuestas que la teoría de Euclides no suministraba, y cuál era la base lógica de esta diferencia.

Lo anteriormente planteado permite reconocer que es anacrónico identificar la propuesta euclidiana contenida en el Libro V de Elementos con la elaboración de Dedekind para los números reales. Esto de ninguna manera niega que sea válido $-\mathrm{y}$ hasta necesario para algunos fines que trascienden la descripción de los hechos históricos- establecer relaciones de la teoría euclidiana con la noción de número real definida por Dedekind, e interpretar aquella teoría a través de construcciones matemáticas modernas $^{11}$; lo que sí niega, es que la lectura moderna de las teorías antiguas

11 La elaboración de Berghout (1974, 1975), no muy citada, ilustra perfectamente la posibilidad y utilidad de lo aquí reseñado. describa satisfactoriamente el hecho histórico. A la luz de esta consideración, se debe reconocer el carácter original de la definición de número real, propuesta por Dedekind, sin que ello obste la potencial intervención de la Definición 5 del Libro $\mathrm{V}$ en la concepción de tal definición de número real.

\section{Frege y la teoría de la proporción}

Una de las referencias que permite una buena aproximación al trabajo de Frege en torno a los números reales es el capítulo 22, "Frege's Theory of real numbers", del libro escrito por Dummett (1991). En la primera parte de tal capítulo se señala que al final de la sección (f) de la Parte III.1 de Grundgesetze, Frege enuncia que se propone definir los números reales, positivos y negativos, como razones de cantidades. Para ello inicialmente expresa las dificultades ligadas a los tratamientos que han intentado, fallidamente, contestar la pregunta sobre qué es una cantidad, para luego señalar que el error en todos los intentos es proponer erróneamente la pregunta. De esta manera, afirma que el concepto esencial no es cantidad, sino tipo de cantidad o, de manera más precisa, dominio cuantitativo (Grössengebiet), y que en lugar de preguntar qué propiedad debe tener un objeto para ser una cantidad, se debe preguntar cuál debe ser la característica de un concepto para que su extensión sea un dominio cuantitativo.

En la segunda parte, se reseña que Frege restringe su trabajo a los dominios cuantitativos no absolutos. Igualmente, que Frege se apropia de una sugeren- 
cia de Gauss ${ }^{12}$ (Gauss, 1863, citado en Dummett, 1991, p. 278) como insumo fundamental de su caracterización de un dominio cuantitativo, y reconoce el carácter central de la necesidad de disponer de una adición y la comparabilidad de las cantidades para la caracterización de los dominios cuantitativos. Atendiendo a ello, y para contestar la pregunta sobre cómo escoger la adición, el orden y los elementos del dominio cuantitativo, Frege considera que un dominio tal debe consistir en permutaciones de un conjunto subyacente y establece que la adición es la composición bajo la cual el dominio es cerrado; así, se tiene que tal dominio debe ser un grupo de permutaciones (ordenado cuando se defina el orden). Sin embargo, la postura puramente estructural, potencialmente subyacente en la anterior afirmación, no es la posición de Frege, pues manifiesta que se requiere de los elementos para constituir las permutaciones y la operación para hacer la adición. Para satisfacer tal necesidad, Frege alude a ilustrar el conjunto subyacente acudiendo al ejemplo de las distancias a lo largo de una recta, pero, según Dummett, con ello, si bien muestra que algunos dominios satisfacen el modelo de Frege, no es capaz de mostrar que todos se pueden caracterizar así; se reconoce así una deficiencia que consiste en tomar por garantía que cada cantidad tiene un valor representable por un real.

12 Gauss señala que los enteros no deben ser asignados a los objetos, sino a las relaciones sobre un conjunto subyacente de objetos con orden lineal discreto, no infinito en ambas direcciones. Una relación tal forma un grupo de permutaciones.
En la cuarta parte ${ }^{13}$, Dummett relata que Frege se ve abocado a probar (usando únicamente recursos lógicos) la existencia de al menos un dominio cuantitativo, para con ello asegurar la existencia de los números reales. En esta dirección, Frege asume como conjunto subyacente aquel conformado por un conjunto de parejas de un entero no negativo y una clase infinita de enteros positivos; estas son reemplazadas por parejas que contienen un número natural y una clase infinita de números naturales (no cero). Para este conjunto define las permutaciones. Sin embargo, observa Dummett, Frege no alcanza la demostración formal de la existencia de un dominio cuantitativo, puesto que las relaciones definidas no son permutaciones; igualmente, Dummett señala la posibilidad y condiciones que podrían haber acompañado el trabajo de Frege para que dicha prueba fuese posible.

La quinta y última parte, describe cómo Frege caracteriza la adición en el conjunto de permutaciones y cómo prueba la satisfacción de tales características. Igualmente, describe la manera en que Frege aborda el problema de introducir la noción de orden, en lo cual interviene la definición de las nociones de clase positival y posteriormente clase positiva, y da cuenta de la manera como Frege caracteriza dicho orden. Adicionalmente, reseña que Frege anuncia la tarea de demostrar la existencia de una

13 No incluimos referencia a la tercera parte, pues esta versa sobre el trabajo de Hölder y su relación con el de Frege y, desde nuestra perspectiva, rompe la secuencia descriptiva del trabajo de este último. 
clase positiva, lo cual abrirá la posibilidad de definir los números reales como razones de cantidades que pertenecen al dominio de la misma clase positiva.

Al final del capítulo, Dummett reseña que si se siguiera la ruta expresada por Frege, en su propósito de definir los reales como razones de cantidades, se tendría la necesidad de asumir la Definición 5 del Libro V de Elementos y que con ello se pudieran definir los números reales como clases de parejas ordenadas de cantidades. Sin embargo, ello conllevaría a que los números reales no serían en sí mismos un dominio de cantidades, como lo demandaba Frege.

Una mirada a lo anteriormente expuesto permite reconocer que la intervención explícita de la teoría euclidiana de la proporción en el trabajo de Frege, no parece ir más allá del uso de la Definición 5 del Libro V, pues toda la elaboración reseñada antes pretende, en últimas, la construcción conceptual matemática de los elementos del dominio de magnitudes sobre los cuales definir la razón (magnitudes para el caso de la propuesta euclidiana). En otras palabras, se puede expresar que Frege advierte, en la teoría euclidiana, la ausencia de una construcción matemática de las magnitudes sobre las cuales definir la razón de magnitudes homogéneas y, en consecuencia, construye unos conjuntos con operaciones y relaciones muy específicas, cuyos elementos puedan relacionarse a través de las razones y de las proporciones, generándose así una idea particular y muy elaborada de número real.

\section{Algunas conclusiones sobre la intervención de la teoría euclidiana de la proporción en la construcción de los números reales}

En esta parte debemos señalar que la teoría euclidiana de la proporción expuesta en el Libro V de Elementos, si bien establece como su piedra angular la Definición 5, es mucho más que esta definición, pues incorpora muchas otras definiciones y varias de las proposiciones no solo se refieren o demuestran con el uso de tal definición. Así, consideramos exagerado afirmar que se usa la teoría euclidiana de la proporción en la creación de una teoría matemática cualquiera, cuando solo se usa una de sus definiciones.

Bajo esta consideración, sería entonces válido -y si se quiere, necesarioestudiar la intervención de cada uno de los elementos constitutivos de una teoría en la construcción o establecimiento de otra, para valorar su efectivo papel. Por ejemplo, con el estudio y explicitación de las diferencias entre las dos "relaciones de orden" que se establecen en una proposición que vincula el "orden" entre magnitudes con el "orden" entre las razones $-\mathrm{o}$ viceversa- $-(v . g$. ., la proposición 10, [Si $A: C<B: C$, entonces $A<B$. Y si $C: A<C: B$, entonces $A>B]$ ), se tendría un argumento adicional para evidenciar la falta de precisión en la transformación de la expresión "Si $n A>m B$ entonces $n C>m D$ " en "Si A/B $>m / n$ entonces $\mathrm{C} /$ $\mathrm{D}>m / n$ " (reseñada antes como parte de la argumentación de quienes sostienen la identidad entre las teorías de Euclides y Dedekind), pues sería claro que la 
primera expresión alude a la relación de "orden" entre magnitudes (representada por el símbolo $<$ ) en tanto que la segunda se establece entre dos razones (y se debería representar con el símbolo - $)^{14}$. Más aún, el análisis debería trascender los elementos constitutivos explícitos, e incluir aquellos que son tácitos y que soportan la teoría misma. Así, por ejemplo, la teoría euclidiana de la proporción atiende a una diferencia básica entre número y magnitud, expresada en el carácter discreto del primero y el carácter continuo de la segunda, entendiendo este último desde la posibilidad de división ad infinitum (en contraste con la imposibilidad de dicha división para los números). Atendiendo a este aspecto, se podría entender con mayor claridad que Dedekind no está trabajando con la misma idea de continuidad, pues de ser así, los números racionales serían para él también continuos.

Un análisis ampliado a un mayor número de elementos constitutivos de la teoría es precisamente lo que creemos que constituye la base argumental que expresa Dedekind en sus cartas a Lipschitz y, en consecuencia, instituyen la racionalidad de la diferencia entre la definición de número real del matemático alemán y la idea de razón $-\mathrm{y}$ proporción-del matemático griego. Un análisis similar es el que seguramente hace parte de las motivaciones que tuvo Frege para desarrollar su trabajo de construcción de los reales, de la manera en que lo intentó, pero en este caso probablemente el análisis mostró no solo el potencial de la teoría, sino también

14 Un argumento similar se podría esgrimir para el caso de la proporción (simbolizada por ::) y la igualdad entre magnitudes (simbolizada por $=$ ). las deficiencias de esta en función de su propósito.

Atendiendo a lo anterior, nos atrevemos a afirmar que no hay una intervención o uso del cuerpo completo de la teoría del Libro V de Elementos, ni en la teoría de los números reales de Dedekind ni en la de Frege; ello no obsta para aseverar que algunos componentes de la teoría euclidiana sí participan (por presencia o déficit) en la constitución de estas teorías.

\section{Utilidad para la educación del profesor de Matemáticas}

Más allá de la utilidad de la historia de las Matemáticas en la educación matemática, queremos referirnos aquí a la potencial utilidad de un asunto histórico específico (la intervención de la teoría euclidiana de la proporción en la construcción del conjunto de los números reales, discutido antes) en el conocimiento del profesor de Matemáticas. Para ello, utilizaremos como marco de referencia las intenciones macro que hemos sintetizado en relación con el estudio de elementos de la historia de las Matemáticas, como parte de la educación de un profesor de Matemáticas (Guacaneme, 2011) y que se refieren a: la transformación de las visiones de la actividad matemática y de los objetos matemáticos, la transformación de la enseñanza de las matemáticas a través de la participación de la historia de las Matemáticas en esta, la potencialidad de la historia como fuente de recursos para la enseñanza, el desarrollo de competencias profesionales y las posibilidades de fortalecer la concepción de la profesión docente en Matemáticas desde la perspectiva histórica. 
Señalemos que el estudio de los aspectos históricos relativos a la teoría euclidiana de la proporción y las teorías de los números reales, implica la familiarización con los aspectos matemáticos de tales teorías. Esto naturalmente se logra a través del desarrollo del estudio de una teoría, actividad matemática legítima y necesaria en la educación de un profesor. Sin embargo, para las teorías en mención, este estudio encuentra escollos poco habituales; uno de ellos se refiere al lenguaje retórico típico de la obra euclidiana, usado también por Dedekind - al menos en el fragmento estudiado (Dedekind, 1968)-, aunque el matemático alemán sí incorpora símbolos como parte del discurso matemático. La experiencia de estudio de unas teorías con estas características textuales, ofrece una excelsa oportunidad para que el profesor pueda comprender, no solo que la escritura de las teorías matemáticas ha evolucionado en el tiempo, sino que las exigencias cognitivas relacionadas con el estudio de una teoría dependen tanto de los aspectos matemáticos de esta, como también de la incorporación o no de un lenguaje simbólico. En suma, esta experiencia tiene el potencial de poner al profesor frente $\mathrm{a}-\mathrm{y}$ dentro de - el problema de los procesos de simbolización como mediadores de la comprensión de las matemáticas.

Otro escollo se refiere a la necesidad de comprender aspectos epistemológicos relativos a los objetos tratados en la teoría, en un contexto de significacióncomprensión que trasciende el contexto personal/cultural coetáneo y que se ubica en otros tiempos y culturas. Así, por ejemplo, quien estudie las teorías tiene que desprenderse, entre otras, de la idea de razón como número o cociente indicado, de la idea de cantidad de magnitud geométrica como medida o número, de la idea de número como punto de la recta, o de la idea que el conjunto de reales es la unión de los conjuntos de los racionales y los irracionales (definidos estos últimos como los reales que no son racionales), para procurar comprender el sentido y los intríngulis de las teorías en cuestión. Lo interesante aquí es que una experiencia tal, aparentemente mediada por el estudio de las matemáticas de otras épocas y culturas, ofrecería la oportunidad al profesor para reflexionar en torno a si el estudio de una teoría no siempre implica, como actividad matemática, la enculturación en una tradición exógena al individuo que realiza el estudio y endógeno al autor de la teoría y a la cultura en la que estuvo inmerso. Además, brinda un contexto para comprender aquello que se ha aludido con la esquiva expresión negociación de significados, cuando se mira el aprendizaje de las matemáticas desde perspectivas socio-semánticas.

Además de la redimensión que respecto de la actividad matemática de estudio de una teoría se pueda lograr a partir de enfrentar los escollos mencionados, la comparación de las teorías ofrece un interesante ámbito para explorar si dentro de las actividades que llevan a cabo los matemáticos, se admite la reformulación o clonación de teorías o de parte de ellas ${ }^{15}$. Este asunto aparentemente cuestionable en el contexto

15 Al respecto no podemos dejar de sugerir el estudio de un artículo que cuestiona si tiene sentido demostrar un mismo teorema en diferentes momentos de la historia (Bagni, 2008). 
de la creación matemática, parece ser natural en el contexto de la comunicación o enseñanza de las matemáticas y puede constituir parte consustancial del quehacer del profesor en su actividad de diseño curricular o de docencia.

Por otra parte, sin lugar a dudas el estudio de la teoría euclidiana de la proporción y su vínculo con las construcciones de los reales, ofrece condiciones propicias para ampliar la visión acerca de conceptos matemáticos (tales como: razón, definición o completitud). De un lado, esta teoría pone de manifiesto la existencia de razones entre objetos matemáticos que no son precisamente números -o siendo redundantes, ni medidas de magnitudes - pero que sí comportan una naturaleza cuantitativa no numérica (usualmente nombrada de manera errónea como cualitativa); colateralmente, ofrece la posibilidad de comprender que para estos objetos se pueden definir operaciones y relaciones, pero que sobre esas relaciones no se puede efectuar una operatividad ${ }^{16}$. De otro lado, la teoría euclidiana brinda la oportunidad de reconocer que una teoría hipotética-deductiva puede contener definiciones que caractericen la existencia de objetos (como la Definición 3 , citada antes), pero que no caracterizan suficientemente a tales objetos y que ello no obsta para la construcción de una robusta y trascendental teoría matemática. Este asunto puede llegar a confrontar la visión que el profesor

16 Desde una aproximación diferente en algún momento presentamos una pregunta relacionada (Guacaneme, 2000) y un colega expresó su postura a propósito de la misma (Obando, 2000). tenga acerca de las exigencias de rigor a las teorías matemáticas y, en especial, a las definiciones implicadas en estas. Asimismo, el estudio de la construcción de los reales vía las cortaduras, ofrece la oportunidad de comprender la diferencia entre la continuidad geométrica y la completitud aritmética, diferencia que sirve de acicate a la formulación de Dedekind. La comprensión que logre el profesor a este respecto definitivamente deberá influir en su conciencia acerca de las estrategias discursivas que utilice en su quehacer docente, sobre todo en aquellas en que se argumenta geométricamente en contextos numéricos o viceversa.

En una dimensión relacionada con el uso de la historia de las Matemáticas en la enseñanza de las Matemáticas, debemos reconocer que, aunque no compartimos la idea del uso de la teoría euclidiana en la construcción histórica de los números reales, no tenemos ningún argumento en contra de explorar la posibilidad de construir escolarmente (seguramente en el ámbito de la educación superior, en los programas de formación inicial de profesores de Matemáticas) el conjunto de los reales a partir de una teoría de la proporción semejante a la euclidiana. Quizá en esta dirección sea conveniente considerar la propuesta de Berghout (1974, 1975) como orientadora de un diseño curricular propicio para construir de manera alternativa los reales, enfatizando en su estructura algebraica y de orden como una aplicación del estudio del álgebra abstracta, que le daría un nuevo sentido a su estudio como parte de la formación matemática de los futuros profesores. 
Finalmente, debemos señalar que ante las implicaciones reseñadas antes, de manera hipotética, respecto de la utilidad del estudio de las relaciones entre la teoría euclidiana de la proporción y las construcciones de los números reales, a favor de la educación del profesor, nos resta invitar a la comunidad de formadores de profesores a investigar empíricamente las bondades y limitaciones de incorporar el estudio de aspectos históricos en sus propuestas curriculares de manera más intensa a como actualmente se hace, al menos en el ámbito colombiano (Torres y Guacaneme, 2011a, 2011b), para así favorecer el conocimiento sobre los procesos y resultados de formación de profesores de Matemáticas y con ello procurar una base firme para un cambio educativo sustancial.

\section{Referencias}

Bagni, G.T. (2008). A Theorem and Its Different Proofs: History, Mathematics Education, and the Semiotic-Cultural Perspective. Canadian Journal of Science, Mathematics and Technology Education, 8 (3), 217-232.

Berghout, R.F. (1974). The Historical Development of Magnitudes, Ratios and Proportions. Australian Mathematics Teacher, 30 (5), 184-196.

Berghout, R.F. (1975). The Historical Development of Magnitudes, Ratios and Proportions. Australian Mathematics Teacher, 31 (2), 66-76.

Corry, L. (1994). La teoría de las proporciones de Eudoxio interpretada por Dedekind. Mathesis. Filosofía e Historia de las Matemáticas, 10 (1), 1-24.

Dedekind, R. (1968). Números irracionales. En J.R. Newman (Ed.), Sigma. El mundo de las Matemáticas (Décima ed.), Vol. 4, pp. 119-128. Barcelona: Ediciones Grijalbo S.A.

Dummett, M. (1991). Frege. Philosophy of Mathematics. Cambridge, Massachusetts: Harvard University Press.

Filep, L. (2003). Proportion Theory in Greek Mathematics. Acta Mathematica Academiae Paedagogicae Nyíregyháziensis, 19, 167-174.

Fine, H. (1917). Ratio, Proportion and Measurement in the Elements of Euclid. The Annals of Mathematics, Second Series, 19 (1), 70-76.

Grattan-Guinness, I. (2004a). History or Heritage? An Important Distinction in Mathematics and for Mathematics Education. American Mathematical Monthly, 111 (1), 1-12.

Grattan-Guinness, I. (2004b). The mathematics of the Past: Distinguishing its History from our Heritage. Historia Matemática, 31 (2), 163-185.

Guacaneme, E.A. (2000). ¿Es posible "sumar" razones? Revista EMA. Investigación e innovación en educación matemática, 5 (3), 284-289.

Guacaneme, E.A. (2011). La historia de las Matemáticas en la educación de un profesor: razones e intenciones. Paper presentado en la XIII Conferencia Interamericana de Educación Matemática, Recife-Brasil.

Guacaneme, E.A. (en prensa). Significados de los conceptos de razón y proporción en el Libro $V$ de Elementos. En O.L. León (Ed.), Énfasis. Libros de los énfasis del Doctorado Interinstitucional en Educación. Bogotá: Universidad Distrital Francisco José de Cladas.

Heath, T.L. (1956). The Thirteen Books of Euclid's Elements (Second ed.). New York: Dover. 
Hill, M.J.M. (1912). Presidential Address on the Theory of Proportion. The Mathematical Gazette, 6 (100), 360368.

Hill, M.J.M. (1928). The Logical Eye and the Mathematical Eye. Their Outlook on Euclid's Theory of Proportion. Presidential Address to the Mathematical Association, 1928. The Mathematical Gazette, 14 (193), 36-56.

Knorr, W. (1992). De exhaución a cortaduras: primeras etapas de la teoría griega de las proporciones. Mathesis. Filosofía e Historia de las Matemáticas, 8, 1-12.

Knorr, W. (2001). The impact of Modern Mathematics on Ancient Mathematics. Revue d'histoire des mathématiques, 7 (1), 121-135. doi: 1262-022X / 1777$568 X$

Obando, G. (2000). Sí, es posible sumar algunas razones. Revista EMA. Investigación e innovación en educación matemática, 6 (1), 69-85.

Puertas, M.L. (1994). Euclides. Elementos. Libros V-IX. Madrid: Editorial Gredos S.A.

Shenitzer, A. (1995). A Topics Course in Mathematics. In F. Swetz, J. Fauvel, O. Bekken, B. Johansson \& V. Katz (Eds.),
Learn from the Masters! (pp. 283-295). Washington, D.C.: The Mathematical Association of America.

Torres, L.A. y Guacaneme, E.A. (2011a). Aproximación a las estrategias curriculares de formación en historia de las Matemáticas en programas de formación inicial de profesores de matemáticas. Paper presentado en el XVIII Congreso Colombiano de Matemáticas, Bucaramanga.

Torres, L.A. y Guacaneme, E.A. (2011b). Caracterización de las estrategias curriculares de formación en historia de las matemáticas en programas de formación inicial de profesores de matemáticas. Paper presentado en él IV Encuentro de programas de formación inicial de profesores de Matemáticas \& V Seminario de Matemática Educativa. Fundamentos de la Matemática Universitaria, Bogotá, Escuela Colombiana de Ingeniería Julio Garavito.

Zubieta, F. (1991). La definición de proporción de Eudoxio. Mathesis. Filosofía e Historia de las Matemáticas, 7, 477-486. 